\title{
Bacteroides nordii
}

National Cancer Institute

\section{Source}

National Cancer Institute. Bacteroides nordii. NCI Thesaurus. Code C86174.

A species of obligately anaerobic, Gram-negative, rod shaped bacteria assigned to the phylum Bacteroidetes. This species is indole positive, but lipase, catalase, urease and nitrate negative, hydrolyzes esculin and gelatin, and is beta-lactamase positive thus resistant to penicillin $\mathrm{G}$, vancomycin, ceftizoxime, cefotetan. B. nordii is commensal and part of the normal human gastrointestinal tract flora, but may become pathogenic in other body regions. 